
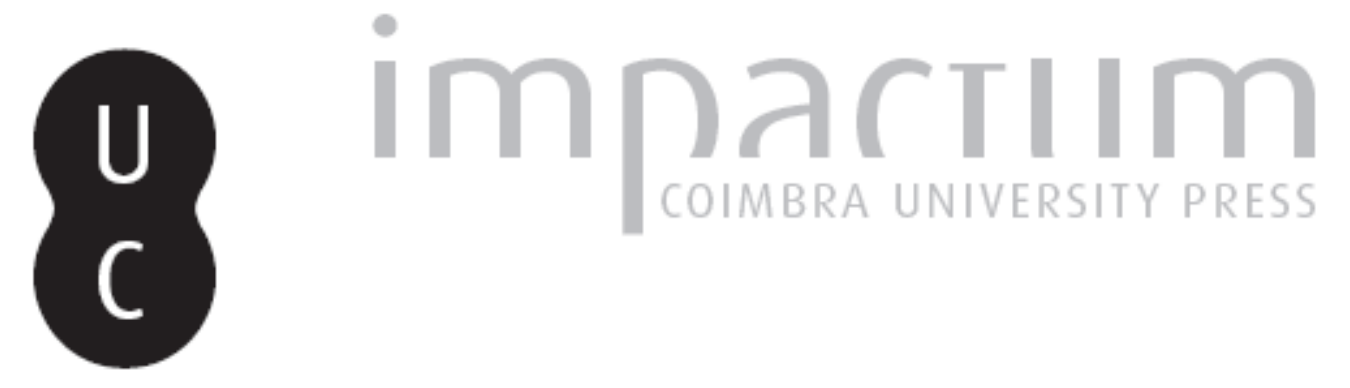

\title{
Deux nouvelles bornes de la voie Ebora-Pax lulia
}

\section{Autor(es): $\quad$ Sillières, Pierre}

Publicado por: Imprensa da Universidade de Coimbra

URL persistente:

URI:http://hdl.handle.net/10316.2/45674

DOI:

DOI:https://dx.doi.org/10.14195/1647-8657_23_4

Accessed : $\quad$ 26-Apr-2023 12:02:16

A navegação consulta e descarregamento dos títulos inseridos nas Bibliotecas Digitais UC Digitalis, UC Pombalina e UC Impactum, pressupõem a aceitação plena e sem reservas dos Termos e Condições de Uso destas Bibliotecas Digitais, disponíveis em https://digitalis.uc.pt/pt-pt/termos.

Conforme exposto nos referidos Termos e Condições de Uso, o descarregamento de títulos de acesso restrito requer uma licença válida de autorização devendo o utilizador aceder ao(s) documento(s) a partir de um endereço de IP da instituição detentora da supramencionada licença.

Ao utilizador é apenas permitido o descarregamento para uso pessoal, pelo que o emprego do(s) título(s) descarregado(s) para outro fim, designadamente comercial, carece de autorização do respetivo autor ou editor da obra.

Na medida em que todas as obras da UC Digitalis se encontram protegidas pelo Código do Direito de Autor e Direitos Conexos e demais legislação aplicável, toda a cópia, parcial ou total, deste documento, nos casos em que é legalmente admitida, deverá conter ou fazer-se acompanhar por este aviso.

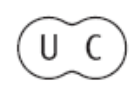


FACULDADE DE LETRAS

INSTITUTO DE ARQUEOLOGIA

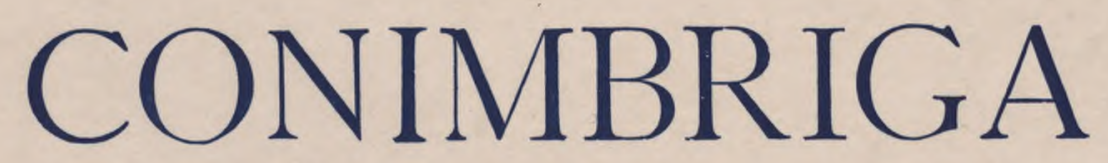

VOLUMEXXIII

UNIVERSIDADE DE COIMBRA

1984 
PIERRE SILLIÈrES

Professeur à l'Université de Toulouse

DEUX NOUVELLES BORNES DE LA VOIE EBORA-PAX IULIA

«Conimbriga», XXIII (1984), p. 55-67

RÉSUMÉ: Au cours de prospections, en vue de reconnaître l'itinéraire de la route romaine de Pax Iulia à Ebora, route qui n'apparaît pas dans l'Itinéraire d'Antonin, deux nouveaux milliaires ont été découverts par l'auteur, à Lindim (Aguiar) e Nossa Senhora de Ares (Viana do Alentejo). L'inscription du milliaire de Lindim est disparue. Le milliaire de Nossa Senhora de Ares, par l'inversion du prénom et du gentilice du César et par l'absence du cognomen, pose des problèmes d'interprétation. L'auter propose son attribution à Crispus, en signalant qu'il ne s'agit là que d'une hypothèse. Le lieu de Nossa Senhora de Ares était probablement au début une mansio qui, par sa position au centre d'un bassin agricole fertile, a pu prospérer.

En Addenda, l'auteur présente la borne qui signalait la mille XI de la même route et restitue un texte qui n'avait pas été correctement interprété jusqu'à présent.

RESUMO : A estrada de Ebora a Pax Iulia não figura no Itinerário de Antonino.

É todavia certa a sua existência e razoavelmente seguro o seu traçado. $\mathrm{O}$ autor identifica dois miliários em Lindim (Aguiar) e Nossa Senhora de Ares (Viana do Alentejo). A inscrição do miliário de Lindim desapareceu. O miliário de Nossa Senhora de Ares, indiscutivelmente in situ, assinalava a milha XVIII, contada a partir de Ebora. As irregularidades do texto tornam apenas hipotética a sua atribuição a Crispus.

Nossa Senhora de Ares corresponde possivelmente a uma estação viária, mansio que se terá convertido num burgo de razoável dimensão. Ficaria provavelmente no limite do território de Ebora.

Em Addenda, o autor apresenta o miliário que assinalava a milha XI da mesma estrada. Não sendo inédito, este miliário, atribuível a Maximino Trácio e seu filho Máximo, não havia sido até agora lido. 
(Página deixada propositadamente em branco) 


\section{DEUX NOUVELLES BORNES DE LA VOIE EBORA - PAX IULIA}

Fax Iulia, colonie romaine et chef-lieu de conventus (x), était reliée à la plupart des grandes agglomérations de la Lusitanie méridionale. En particulier, une voie la mettait en relation avec Ebora, une des plus importantes cités de cette région ${ }^{(2)}$.

$\mathrm{Au}$ cours de prospections, en vue de reconnaître l'itinéraire précis de cette route romaine, deux nouveaux milliaires ont été découverts: l'un est malheureusement brisé et son inscription a disparu; l'autre, en revanche, est intact et constitue donc un nouveau document routier fort intéressant.

Cet itinéraire n'apparaît pas clairement dans l'Itinéraire (TAntonin (3) car ce médiocre guide routier présent en désordre les stations $\mathrm{du}$ long périple menant $\mathrm{d}$ Esuri (Castro Marim) à Arucci (Aroche) par Salada (Alcácer do Sal) et Ebora; en particulier, Pax Iulia est oubliée entre Ebora et Serpa (Serpa). Aussi n'a-t-il pas été retenu dans la carte des voies du Portugal romano $\left({ }^{4}\right)$. Pourtant, ses vestiges archéologiques sont nombreux, surtout les bornes milliaires.

P) Sur cette Colonia civium Romanorum, voir A. Tovar, Iberische Landeshunde, 2, Lusitanien, Baden-Baden, 1976 (= Tovar, Lusitanien),

p. 211-212.

(2) Cf. T ovar, Lusitanien, p. 217-218.

(3) It. Ant., 425,6-427,3.

(4) J. Alarcão, Portugal Romano, Lisbonne, 1974, p. 67. En revanche, elle est portée sur la carte de H. Kiepert, CIL, II, suppl., in fine, ainsi que sur celle d'E. SaAvedra, Discursos leídos ante la Real Academia de la Historia, Madrid, 1862, carte in fine.

Conimbriga, 23 (1984), 55-67 


\section{1) Les milliaires connus}

Resende, le célèbre épigraphiste de la Renaissance, avait déjà attiré l'attention sur cette route romaine en mentionnant ses ponts et ses bornes $\left(^{5}\right)$. Toutefois, il ne précisa pas leur position et ne rapporta qu'une inscription d'Hadrien $\left(^{6}\right)$. Mais ces renseignements ne furent pas exploités par Hübner qui ne mentione entre Salacia et Pax Iulia que deux bornes perdues dont il ne connaît pas du tout la provenance et qui ne correspondent pas à celle de Resende $\left({ }^{7}\right)$.

Aussi, jusqu'à une date assez récente, ne savait-on à peu près rien de cette voie, jusqu'à ce qu'un archéologue portugais s'intéresse aux voies de son pays $\left({ }^{8}\right)$. Le grand mérite de M. Saa est d'avoir recherché sur le terrain les vestiges des routes antiques ${ }^{9}$ ). Par exemple, ses prospections lui ont permis de voir au moins une douzaine de bornes pour la voie qui nous intéresse ici. En voici une rapide rappel:

- Fonte do Seixo: 1 fragment de milliaire anépigraphe.

- Entre Zambujeira et Magalhào: 4 milliaires dont les inscriptions on disparu par érosion du granite; connu sous le nom de «Marco dos Diabos», l'un d'entre eux est encore dressé au bord du chemin antique $\left({ }^{10}\right)$.

- Gué de Camoeira sur le Xarrama: 5 ou 6 bornes cassées servant de «pas japonais», pour permettre aux piétons de franchir la rivière à pied sec. Un seul, qui, semble-t-il, a disparu

(5) «... Item super Exarramam, Murtariam et Odivellam fluvios pontium dirutorum hic sublicia illic pilae, pilarum bases. Litterae in columnis intercidere...»-A. DE RESENDE, De Antiquitatibus Lusitaniae, Coimbra 1970, I p. 220-221.

$\left(^{6}\right)$ «... In una ... potui legere ... Imperatori Caesari Nervae Nepoti Trajanus Hadrianus Consul tercium...», ibid.

( $\left.{ }^{7}\right)$ CIL, II, 4629 et 4630.

(8) M. SAA, as grandes vias da Lusitânia, 6 vol., Lisbonne, 1956-1964; pour l'étude intéressant la voie Ebora-Pax Iulia, voir le tome IV, 1963, p. 234-269 (= SAA, Vias).

$\left(^{9}\right)$ Aussi ne lui fera-t-on pas grief de ses localisations de villes antiques qui sont parfois assez fantaisistes.

(10) SAA, Vias, p. 251 et fig. 14. 
récemment $(\mathrm{n})$, portait une inscription incomplètement lue et attribuée à Hadrien probablement à tort $\left({ }^{*}\right.$ il 12$)$. Ces morceaux de bornes proviennent de la voie romaine qui passe à un demi-kilomètre de là.

- Nossa Senhora de Ares: milliaire dressé à l'est de cette église de pèlerinage voisine de Viana do Alentejo, à l'endroit où l'avait photographié M. Saa $\left({ }^{13}\right)$. On ne peut déchiffrer que le nombre XVII, indiquant au bas de la pierre la distance mesurée depuis Évora, et un $\mathrm{O}$ appartenent au texte, un peu au-dessus. Néanmoins, une étude plus précise, avec estampage, permettrait peut-être d'obtenir de meilleurs résultats.

\section{2) Les nouveaux milliaires}

— Le milliaire de Lindim (Aguiar) (pi. I)

Devant la ferme de Lindim, située à $3 \mathrm{~km}$ au sud-ouest d'Aguiar ${ }^{(14)}$, est conservée la partie inférieure d'une borne milliaire, composée de sa base et du début du fût.

Matériau: granite.

Dimensions: hauteur totale: $98 \mathrm{~cm}$ dont base prismatique haute de $46 \mathrm{~cm}$ et fût $52 \mathrm{~cm}$; diamètre du fût: $48 \mathrm{~cm}$.

Inscription: disparue.

- Le milliaire de Nossa Senhora de Ares (Viana do Alentejo) (pi. II et III)

A une centaine de mètres au nord de l'église, à la limite ouest du site archéologique, est abandonné au sol un milliaire intact,

(u) Selon le témoignage d'un gardien de troupeau, il aurait été enlevé, il y a deux ou trois ans, pour une destination inconnue.

(12) SAA, Vias, p. 239 et fig. 16; en effet, le surnom Fel(ix) ne fut porté par les empereurs qu'à partir de Commode; aussi préfèrerions-nous proposer Caracalla, qui prit un grand soin des voies de la Péninsule ibérique; le texte lu par M. Saa était: V / VSO / FEL / XIY / PP / FEC.

(13) SAA, Vias, p. 256, fig. 19.

(14) Coordonnées géographiques de la ferme: $7^{\circ} 59^{\prime} 45^{\prime \prime}-38^{\circ} 21^{\prime} 50^{\prime \prime}$. 
au milieu d'un tas de pierres, de blocs antiques, de tegulae et de fragments d'opus signinum.

Matériau: calcaire jaunâtre.

Forme: borne grossièrement cylindrique avec une base élargie et aplatie.

Dimensions: hauteur totale $158 \mathrm{~cm}$ dont $40 \mathrm{~cm}$ pour la base et 118 pour le fût; diamètre $32 \mathrm{~cm}$.

Champ épigraphique: $59 \times 30 \mathrm{~cm}$.
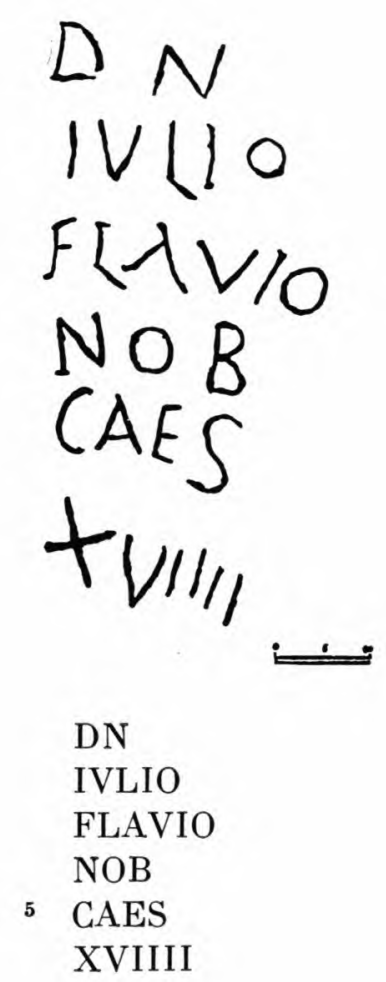

D(omino) N(ostro), / Iiilio / Flavio, | nob(ilissimo) / 5 Caes(ari) / \{milia passum) xviiii.

Hauteur des lettres: 1. 1: 6; 1. 2: 4-7; 1. 3: 5-7; 1. 4: 4,5-7,5; 1. 5: 6-8; 1. 6: 5-8. Espacements: 1: 2; 2: 3; 3: 2,5-3,5; 4: $1-3 ; 5: 4,5-6$.

Conimbriga, 23 (1984), 55-67 
Malgré un effort d'alignement à gauche, la gravure de ce texte est bien maladroite: le lapicide n'a pas su conserver l'horizontalité des lignes qui, toutes, penchent vers le bas sur le côté droit; en outre, les dimensions des lettres varient considérablement dans une même ligne et les espacements sont aussi fort différents. Enfin, l'écriture n'a aucune régularité, certaines lettres étant fortement influencées par la cursive, en particulier les L et le A de la troisième ligne: il est enfin remarquable que la forme d'une même lettre peut varier, par exemple celle des A de Flavio et de Caes.

Néanmoins, la gravure est assez profonde pour que le déchiffrement de cette inscription n'offre aucune difficulté. Mais cela ne veut pas dire que ce texte soit d'interprétation facile. En effet, il présente deux graves anomalies: la première réside dans l'ordre des éléments du nom Iulius Flavius car il est inhabituel et tout à fait incorrect de placer Iulius avant Flavius; au contraire, on trouve toujours le gentilice Flavius, utilisé comme prénom par les princes de la dynastie constantinienne, en premier lieu et précédant le gentilice Iulius; cette inversion paraît un hapax $\left({ }^{15}\right)$ et met en évidence l'ignorance de 1 'ordinator qui ne devait pas avoir une idée bien claire de la titulature impériale; la deuxième anomalie, bien plus grave d'ailleurs, est l'absence de cognomen, car elle nous condamne à l'incertitude pour l'identification du César honoré ici.

On connaît, en effet, plusieurs princes s'appelant Flavius Iulius et qui reçurent le titre de César: ce sont les trois fils de Constantin le Grand, Flavius Iulius Crispus $\left({ }^{16}\right)$, Flavius Iulius Constantius $\left({ }^{17}\right)$ et Flavius Iulius Constans $\left({ }^{18}\right)$, auxquels il faut ajouter leur cousin Flavius Iulius Delmatius $\left({ }^{19}\right)$. Il est donc très difficile de faire un choix $\left({ }^{20}\right)$ et nous ne pourrons proposer qu'une identification très hypothétique.

(15) La consultation des instruments épigraphiques habituels, CIL, $E E, A E, D E$, n'apporte aucun autre exemple.

(16) DE, II, p. 653-655; Crispus fut César de 317 à 326.

(17) $D E$, II, p. 668-676; Constance fut César de 324à 337.

(18) DE, II, p. 627-631; Constant fut César de 333 à 337.

j19) DE, II, p. 667-668; Delmace fut César de 335 à 337.

${ }^{20}$ Il parait néanmoinsnormal de négliger Flavius Iulius Maiorianus (457-461).

Conimbriga, 23 (1984), 55-67 
Dans ce but, la seule démarche est, semble-t-il, de rechercher lequel de ces princes fut le plus fréquemment honoré dans la Péninsule Ibérique lorsqu'il était César. En fait, aucun ne le fut très fréquentement, mais Crispus l'emporte nettement puisque du fils aîné de Constantin on connaît déjà cinq bornes milliaires $\left({ }^{21}\right)$ et, probablement, une dédicace pure $\left({ }^{22}\right)$. Ensuite, vient Delmace, au nom duquel, malgré la brièveté de son césarat, on grava quelques milliaires, deux peut-être $\left({ }^{23}\right)$. De Constance César, on connaît une borne sur laquelle il est honoré avec son ainé Constantin (24) et une dédicace $\left({ }^{25}\right)$. Enfin, il n'y a aucune borne connue au nom de Constant César qui n'apparaît que sur une dédicace de Chaves ${ }^{(26)}$.

Aussi, en répétant qu'il ne s'agit là que d'une pure hypothèse fondée sur les inscriptions connues aujourd'hui, donc, en partie, sur le hasard, proposons-nous d'attribuer cette borne à Crispus, le fils aîné de Constantin. Le rôle que paraît lui avoir réservé son père dans les affaires d'Occident $\left({ }^{27}\right)$, peut expliquer une popularité plus grande que celle de ses frères.

La dernière ligne du texte confirme l'indication de l'autre milliaire de l'église d'Ares: comme le proposait M. Saa, c'est bien d'Ebora que les milles étaient comptés $\left({ }^{28}\right)$. En effet, l'itinéraire de la voie entre Évora et Ares étant assuré, non seulement par les nombreuses bornes qui la jalonnent, mais encore grâce au vieux nom d "Estrada dos Diabos qui la désigne sur tout ce trajet, il est facile de vérifier les distances: par mesure au curvimètre le long

(21) CIL, II, 4764 = ILER, 1868; L. WICKERT, De nonnullis milliariis Bracarensibus, dans Homenagem a Martins Sarmento, 1933, p. 446; AE, 1976, 282 d; AE, 1977, 376 et 436.

(22) CIL, II, 4107.

(23) EE, VIII, 223a et probablement, Rodriguez Colmenero, La red viaria romana del Sudeste de Galicia, Valladolid, 1976, p. 110, n. ${ }^{\circ} 49$.

(24) CIL, II, 4700.

(25) $C I L$, II, $4108=I L E R, 1234$.

(26) ILER, 1232.

(27) Il reçut le commandement nominal des troupes défendant le limes contre les Francs et les Alamans en 320, puis de la flotte envoyée contre Licinius en 324.

(28) SAA, Vias, p. 249. 
de cette Estrada dos Diabos, on constate que l'église d'Ares est à $28 \mathrm{~km}$ d'Évora, c'est-à-dire exactement à 19 milles.

Aussi, peut-on en conclure que le Caput Viae était bien Ebora et que le milliaire nouveau portant la distance de XVIIII milles était dressé à Ares. En revanche, l'autre borne, celle qui indique seulement XVII milles, n'est pas in situ près de l'église; elle était originellement dressée à $3 \mathrm{~km}$ de là, en direction d'Évora, c'est-à-dire aux alentours de Samarra $\left({ }^{29}\right)$.

$\mathrm{Au}$ total, on connaît donc aujourd'hui l'existence d'une quinzaine de bornes milliaires entre Évora et Viana do Alentejo. Ce nombre élevé est fort étonnant alors qu'il n'en subsiste pas une seule entre Viana et Beja, c'est-à-dire sur une distance légèrement supérieure $\left({ }^{30}\right)$. Le hasard des découvertes ne peut expliquer une telle disparité.

En réalité, il n'y eut sans doute pas de bornage régulier entre Viana et Beja. Cette explication peut paraître singulière; elle s'explique pourtant par l'organisation des travaux routiers dans l'Empire romain. Certes, les constructions et les réfections de la voirie relevaient de l'empereur, mais les travaux eux-mêmes étaient le plus souvent effectués dans le cadre de la civitas, puisque les dépenses incombaient généralement aux propriétaires riverains $\left({ }^{31}\right)$. Le conseil des décurions et les édiles municipaux avaient donc la charge des chantiers sur le territoire de leur cité. La conséquence était que les aménagements ou les restaurations s'arrêtaient à la frontière de celle-ci. Voilà pourquoi il n'y eut de bornage que dans la cité d'Ebora.

\section{1) La frontière entre les deux cités}

Cette constatation permet de proposer une délimitation approximative entre les territoires à'Ebora et de Pax Iulia. En effet,

$\left.{ }^{29}\right)$ Coordonnées géographiques de la ferme de Samarra: $7^{\circ} 59^{\prime} 45^{\prime \prime}$ $-38^{\circ} 22^{\prime} 48^{\prime \prime}$.

${ }^{(30)}$ Il y a $29 \mathrm{~km}$ d'Évora à Ares et environ 40 de cet endroit à Beja.

(31) À ce propos du financemente des travaux routiers dans l'Empire romain, on se reportera à T. PEKARY, Untersuchungen zu den rõmischen Reichsstrassen, Bonn, 1968, p. 113-115.

Conimbriga, 23 (1984), 55-67 
le dernier des milliaires de granite, de la même série que ceux d'Aguiar et de Camoeira, porte le nombre XVII, et celui que nous venons de publier indique la distance de XVIIII milles, mesurée également depuis Évora.

Au-delà, il n'y a plus aucune borne. Aussi, à notre avis, la frontière entre les cités de Pax Iulia et d'Ebora devait passer dans cette zone. On remarquera d'ailleurs que la limite actuelle entre les districts de Beja et d'Évora est aussi légèrement au sud de Nossa Senhora de Ares.

\section{2) Vagglomération antique de Ares}

Une courte exploration des alentours de l'église suffit à convaincre de l'importance de ce gisement archéologique occupant peut-être une dizaine d'hectares et conservant des vestiges de grands bâtiments. Il a en effet fourni de nombreux vestiges et sa nécropole a, en partie, été fouillée ( $\left.{ }^{32}\right)$. Plusieurs inscriptions en proviennent, non seulement celles qui sont prises dans les piliers de l'entrée de la clôture de l'église ( $\left.{ }^{33}\right)$, mais aussi probablement celles que le $C I L$, II, attribue à Alvito ( $\left.{ }^{34}\right)$.

Il s'agit donc d'une agglomération antique de quelque importance. Mais de quel genre ? A titre d'hypothèse, on proposera d'y voir un bourg routier qui aurait réussi. Au début simple mansio sur la route Ebora-Pax Iulia, sa position, au centre d'un bassin agricole fertile, lui permit sans doute de se développer et de prospérer.

Voilà donc une voie romaine qui sort de l'ombre où l'avait plongée VItinéraire d'Antonin; grâce à sa richesse en milliaires, son tracé ne fait plus de doute, au moins entre Évora et Viana do Alentejo. Mais il y a encore beaucoup mieux à faire: la fouille du grand site archéologique de Ares serait d'un grand intérêt car elle mettrait au jour un bel exemple de bourg routier.

(32) F. Alves Pereira, Antiguidades de Vianna do Alentejo, dans AP, 9, 1904, p. 271-296 et 10, 1905, p. 16-28.

(33) CIL, II, 88 et 89.

H CIL, II, 87, 90, 91 et 92 .

Conimbriga, 23 (1984), 55-67 


\section{ADDENDA}

Cet article était déjà sous presse lorsque nous avons appris par notre ami Monsieur José d'Encarnaçâo que la borne milliaire de Camoeira n'était pas perdue mais qu'elle avait été déposée à Évora dans les entrepôts du Service des Travaux publics où Madame Maria Manuela Borges Fernandes, qui avait assuré le sauvetage de ce précieux document épigraphique, nous a permis de l'étudier à loisir et de rectifier l'attribution proposée à la note 12. Qu'elle veuille bien trouver ici l'expression de notre vive reconnaissance.

Lieu de conservation actuel: Évora, Direcção de Estradas.

Lieu d'invention: Camoeira (5,5 km au nord d'Aguiar, sur la rive droite du Xarrama).

Forme: Milliaire en granite rose jaunâtre, conservé intégralement. Hauteur totale: $260 \mathrm{~cm}$ ? Base: hauteur $70 \mathrm{~cm}$ ? arêtes $60 \mathrm{~cm}$. Fût: hauteur $194 \mathrm{~cm}$, diamètre à l'extrémité supérieure $50 \mathrm{~cm}$, à la base $62 \mathrm{~cm}$.

Champ épigraphique: 98 x 143.

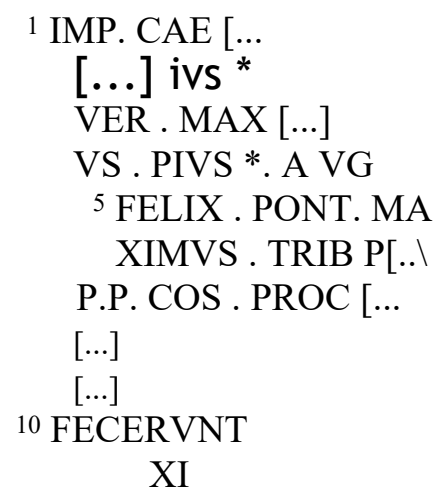


Imp(erator) Cae[s(ar) / C(aius) Iui] ius / Ver(us) Maxlimirillus, Pius, Aug(ustus), / FCZZÆ, pont(ifex) majximus, tribunicia) p[ot(estate)] I, p(ater) p(atriae), co(n)s(ul), wZ) /

C(aius) Iulius / Maximus nob(ilissimus) Caes(ar)] / fecerunt, I [milia passuum) xi.

Hauteur des lettres: Lia 10: 11-12 cm; 1. 11: $14 \mathrm{~cm}$; espacements: 2-3 cm.

Bibliographie: SAA, Fias, p. 239 et fig. 16; Túlio EsPanca, Inventário Artístico de Portugal - VII - Concelho de Évora, Lisboa, 1966, p. 353; texte fort incomplet puisque les premières lettres de chaque ligne, seulement, avaient pu être lues. «Diário do Sul», Évora, 6 Juin 1983 (notice et photo).

Inscription assez peu soignée: certes, elle est constituée de lettres profondément gravées mais cette écriture de type monumental présente des caractères de dimension irrégulière et l'horizontalité des lignes est rarement observée. En outre Vordinatio est fort maladroite: pas de véritable alignement à gauche, et, surtout, mauvaises coupures des mots (MAXIMIN/VS et MA/XIMVS); enfin, espacements irréguliers entre les lettres d'un même mot, par exemple pour AVG, TRIB, PROC et surtout, FECERVNT. Quant au texte lui-même l'ordre anormal des surnoms impériaux, Pius, Augustus, Felix, choque. Mais cette inscription a surtout été défigurée par un martelage des lignes 2 et 3 puis 8 et 9 .

Cette borne paraît néanmoins devoir être attibuée à Maximin le Thrace et à son fils Maxime. Plusieurs arguments y incitent: surtout, en premier lieu, la présence très nette des lettres VER qui n'ont pas été martelées à la ligne 3 et les restes d'autres lettres ayant subi la martelage, IVS à la ligne 2 et MAX à la ligne 3; ensuite, la longueur convenable des noms de Maximin et de Maxime pour les espaces martelés; troisièment, l'existence en Lusitanie d'un autre milliaire de ces mêmes princes, pierre dont le texte se termine également par fecerunt (CIL II, 4649); enfin, un argument supplémentaire à considérer est que les autres attributions possibles - en négligeant les traces IVS et MAX à Caracalla et Geta d'une part, à Macrin et Diaduménien d'autre 
part, sont invraisemblables, ces princes paraissant s'être désintéressé totalement des routes de la Péninsule ibérique.

Aussi proposons-nous de dater la pose de cette borne milliaire des années 236-238, à un moment qui ne peut être mieux précisé puisque aucune indication n'est conservée pour les puissances tribuniciennes et que l'on ne doit pas tirer de conclusion assurée de l'absence de nombre après la mention du consulat.

Cette pierre avait été dressée à 11 milles d'Évora, ce qui, en effet, conduit aux alentours de Camoeira. 
PL. I

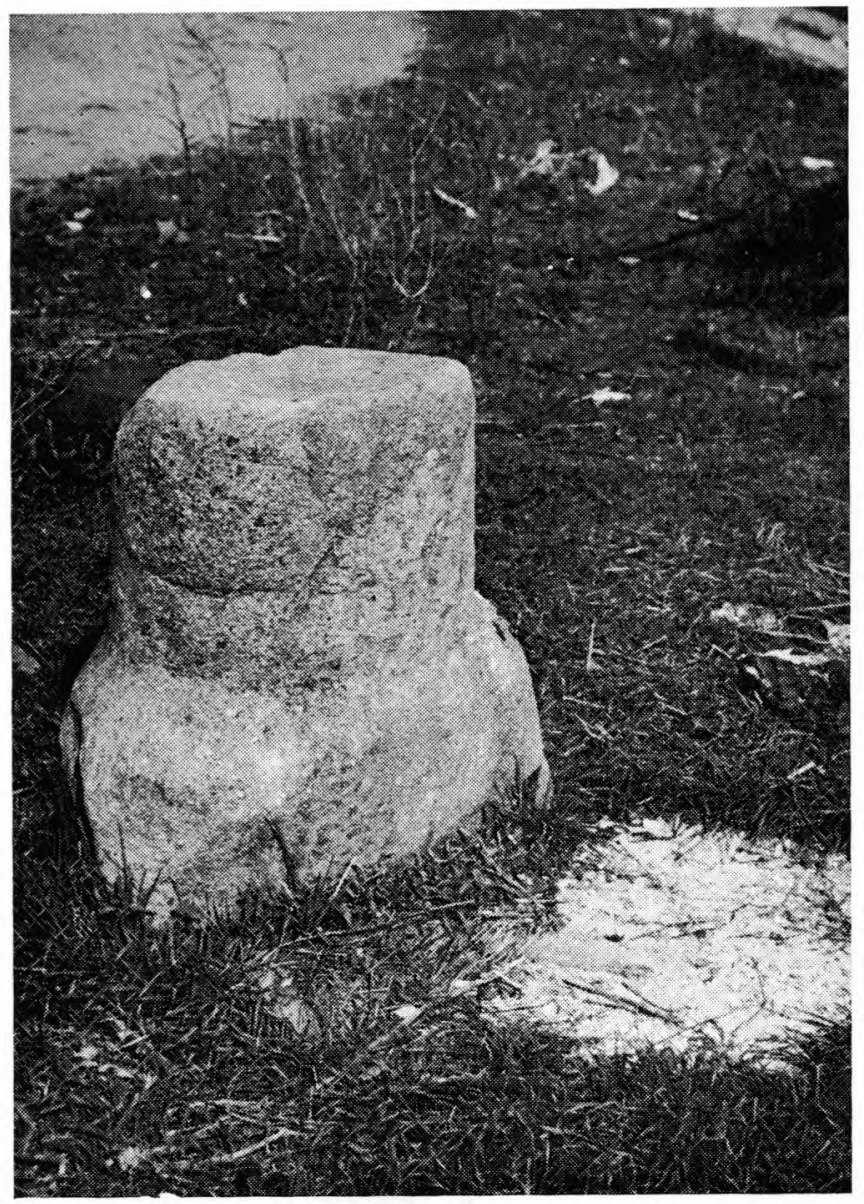


PL. II

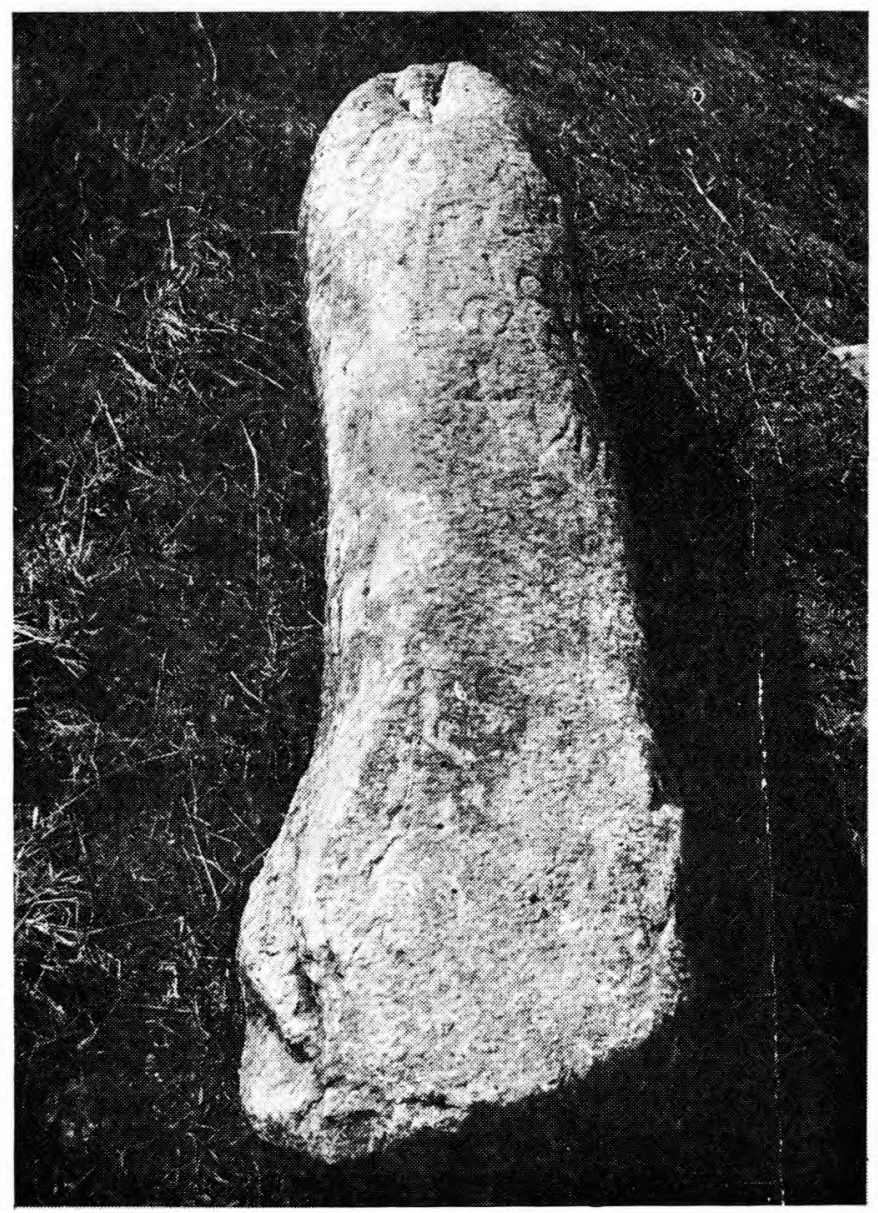


PL. Ill

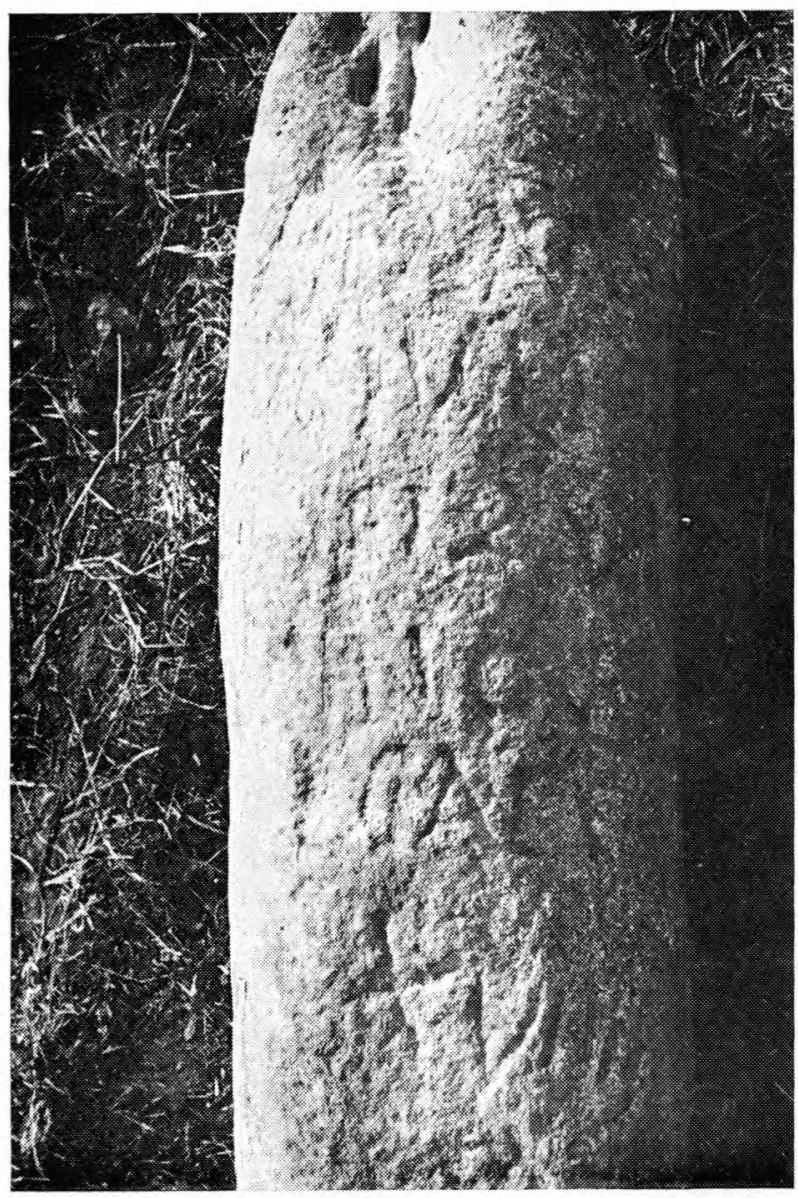


PL. IV

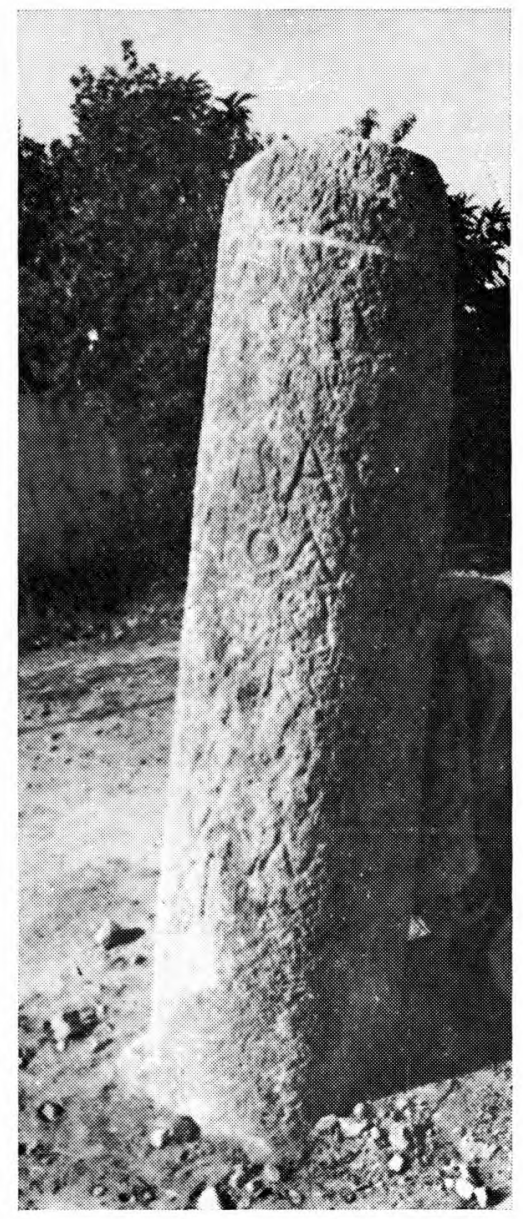


PL. Y

$$
\text { IMP.CA' }
$$

VER./NAY

VS.PIVS.AVC

EELIX.PO NT:M

XINAVS.TRIBP

P.P.COS.PROC

FECER $V N T$

$X I$

Ln 